\title{
Improving Therapeutic Interventions of Schizophrenia with Advances in Stem Cell Technology
}

\author{
José Andrés Espejo Oltra \\ School of Experimental Sciences, Valencia Catholic University Saint Vincent Martyr, Valencia, Spain
}

\begin{abstract}
Although historic documents posit schizophrenia to the beginnings of mankind, its diagnosis remains poorly defined, currently relying on unspecific clinical symptoms; and controversies still maintain its origin under intense debate. This review aimed at quantitatively assessing the preferential forefronts of clinical trials towards the treatment of schizophrenia from inception till present, according to clinicaltrials.gov database registry. Towards that end study status and study phase classifications were used as criteria for progress in the field. Study groups by sex and age together with countries and organisms involved in the studies were used as indicators of the populations studied and as evidence of main promoter institutions, in both, pharmacological and drug-free protocols. The findings clearly show a decline of active clinical research with small synthetic compounds and limited numbers of novel initiatives, mostly based on drug-free alternatives with expected reduced secondary effects. A paucity of sex- and age-oriented designs is detected, and it is proposed that future clinical trials should set their basis on data obtained from patient-derived induced pluripotent stem cells, brain organoid systems and human brain circuitry platforms. Only individual precision medical approaches may turn effective for the treatment of this complex and highly incapacitating disease.
\end{abstract}

KEY WORDS: Schizophrenia; Pragmatic clinical trials; Therapy; Biomarkers; Stem cell research; Neurogenesis.

\section{INTRODUCTION}

Schizophrenia is a complex mental disorder typically manifesting multiple neuropsychiatric symptoms, such as hallucinations, delusions and disorganized speech. Affected individuals show reduced motivation, social withdrawal and cognitive deficits [1]. The illness quite strikingly impacts the quality of life of the affected individuals, their families and society. With only $8 \%$ employed, the disease represents a considerable economic burden with approximate costs of 11.8 billion pounds in United Kingdom and 155.7 billion US dollars in the US according to recent studies $[2,3]$.

The risk for developing schizophrenia during lifetime is set at $1-1.5 \%$ globally, and thus, the most prevalent dis-

\footnotetext{
Received: April 7, 2020 / Revised: May 13, 2020

Accepted: May 17, 2020

Address for correspondence: José Andrés Espejo Oltra

School of Experimental Sciences, Valencia Catholic University

Saint Vincent Martyr, c/Guillem de Castro, 94, Valencia 46001, Spain

E-mail: jose.espejo.oltra@gmail.com

ORCID: https://orcid.org/0000-0002-8036-3260
}

ease among major psychoses, frequently manifesting symptoms in early adulthood [4]. In addition, the lifetime expectancy of schizophrenia patients is reduced by 15 to 20 years compared to people not suffering any severe mental illness highlighting the life-threatening character of the disease $[2,3]$.

The etiology of schizophrenia is so amply complex that its causes are not yet fully understood. Both, genetic and environmental factors seem to constitute the main players in the occurrence of the disease. About $40 \%$ of genes linked to schizophrenia deal with the response of the brain to infections [5]. As per environmental triggers, exposure to inflammation or acute stressors in utero, on one side, and perinatal brain injury and/or chronic cannabis consumption, or other drug abuse, on another, are believed to be determinant for disease onset [6]. In any case it seems that dysregulation of brain dopamine pathways is a common underlying cause of symptoms and thus the target of many drug-based therapies [7]. Glutamatergic deficient neurotransmission has also obtained attention as a key defect in schizophrenia [8]. In addition, a subgroup

(c) This is an Open-Access article distributed under the terms of the Creative Commons Attribution Non-Commercial License (http://creativecommons.org/licenses/by-nc/4.0) which permits unrestricted non-commercial use, distribution, and reproduction in any medium, provided the original work is properly cited. 
of about 30 to $50 \%$ of patients report low levels of inflammation with increased production of proinflammatory cytokines and $C$ reactive protein $[9,10]$, perhaps due to microglia "priming" as a result of early life exposure to inflammatory agents. Due to patient heterogeneity the diagnosis of schizophrenia at present relies solely on clinical manifestations [8], and therefore available treatments are unspecific and varied.

An increasing emphasis for early intervention of individuals manifesting psychotic symptoms has been noticed in recent years aimed at promoting recovery and preventing functional detriments $[11,12]$.

Among top recommendations for people between 15and 17-years old presenting schizophrenia or recurrent psychosis by the National Institute for Health and Care Excellence guidelines (Department of Health of the United Kingdom) [13] is the anti-psychotic aripiprazole, also prescribed for the treatment of bipolar disorder, major depressive disorder, autism and even for tic disorders. Its mode of action is related to dopamine and serotonin signaling, and common side effects include weight gain, vomiting, sleepiness, dizziness and constipation. Serious secondary reactions include anaphylaxis (serious allergic reactions that might lead to death), tardive dyskinesia (characterized by involuntary repetitive movements or "jerking" movements) and neuroleptic malignant syndrome (characterized by muscle rigidity, fever, confusion, fast heart rate and sweating that can be life-threatening).

With the aim of assessing past and new efforts towards the clinical treatment of schizophrenia, this review examines what have preferential forefronts of clinical trials (CTs) been from inception till present. Classification of study status and study phase are used as criteria of progress in the field. Study groups by sex and age together with countries and organisms involved in the studies are evaluated as indicators of populations studied and main promoters of both, pharmacological and drug-free methods to treat the disease. The review concludes presenting recent key advances in the field of stem cell biology as novel tools to revolutionize future forefronts of schizophrenia treatment research. It is expected that they will set the basis for the prospective design of clinical trials with an individualized perspective.

\section{PAST CLINICAL TRIALS FOR THE TREATMENT OF SCHIZOPHRENIA OVERWEIGHS NEW INITIATIVES}

A search of registered clinical trials (Clinical trials webpage, 2019) [14], using the terms: "schizophrenia" in the disease option AND "drug" OR "treatment" as additional terms, yielded a total of 3,086 studies. CT were further classified in relation to: study status, eligibility criteria of participants (age and sex), study type, study phase, funder type and geographic location for each one of the searches above mentioned. Findings were expressed as percentages of the overall data.

Among the 3,086 CTs performed towards the treatment of schizophrenia, 1,929 of them, representing $>60 \%$ corresponded to completed studies and only 654 studies ( $21 \%$ ) corresponded to active studies (including the categories for recruiting; enrolling by invitation; active, not recruiting and not yet recruiting) (Fig. 1A). This indicates that past research on schizophrenia therapeutic options overweighs current clinical research efforts.

Among the 1,929 studies completed, almost 50\% $(1,483 / 3,086)$ did not have a study phase annotated; and the rest of the phases oscillated between $11-15 \%$ for each of them with lowest numbers corresponding to early phase and phase I studies (Fig. 1B), again supporting a situation of reduced new forefronts compared to more advanced, old research.

Interventional studies were found to be prevalent (85\%) over observational study designs (15\%). Although no sex bias was notorious, a slight predominance of the male participants in the studies was noticed, suggesting the possibility of male-directed studies. Review of age inclusion criteria showed that adults from ages 18 to over 65 participated in most studies, while only $14 \%$ of the studies included patients of pediatric age $(<18)$ (Supplementary Table 1; available online).

Regarding funding resources, it was found that main investors correspond to Universities and other, with almost $50 \%$ of the invested money, closely followed by Industry. The lowest investment is attributed to Federal resources (National Institutes of Health and other, representing together only around $15 \%$ of them) (Fig. 1C; Supplementary Table 1).

It must be noted that the sections of participants by sex and age add to over $100 \%$ of the total number of studies 
A
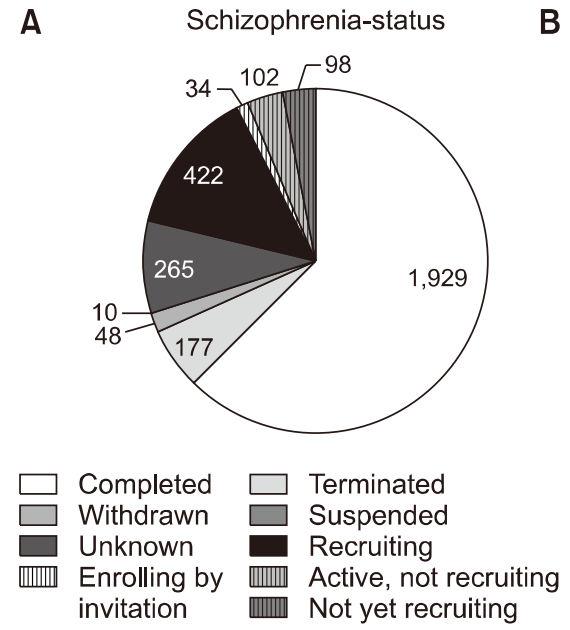

D Schizophrenia-completed

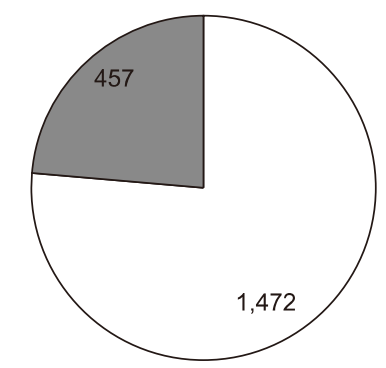

W/out results $\square$ With results

\section{$\mathrm{E}$}

B
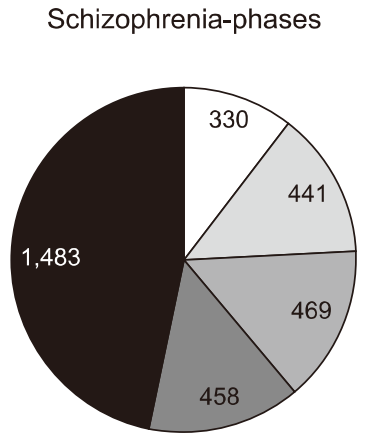

Early/phase I Phase III

N/A

\section{Schizophrenia-completed phases}

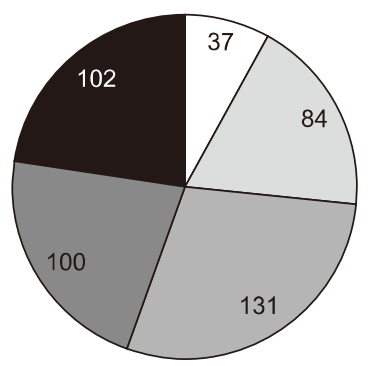

$\square$ Phases early/phase I
Phases phase III

Phases N/A

\section{C \\ Schizophrenia-funding}

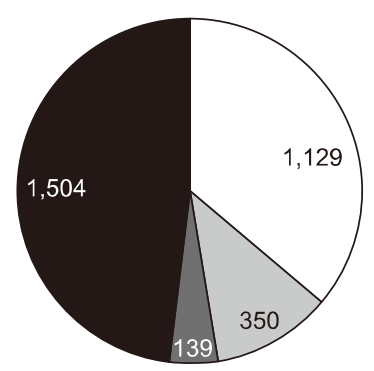
$\square$ Industry
$\begin{aligned} & \text { Other federal } \\ & \text { agencies }\end{aligned}$ $\begin{aligned} & \text { UnH } \\ & \text { Universities/other }\end{aligned}$

\section{F Schizophrenia-completed funding}
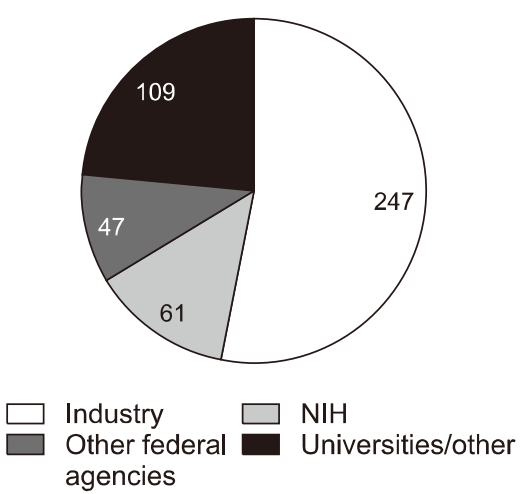

Fig. 1. Clinical trials (CTs) for the study of schizophrenia. Classified according to study status as completed, terminated, withdrawn, suspended, on unknown status, recruiting patients (ongoing), enrolling by invitation (ongoing), active, not recruiting (ongoing), not yet recruiting (registered, not active) at the time of consultation (A); according to study phase, catalogued as Early phase/Phase I, as a single category, Phase II, Phase III, and Phase IV (B). N/A corresponds to trials that have not been catalogued by study phase. And according to funding resources as coming from industry, $\mathrm{NIH}$, Federal agencies other than NIH, and Universities or other institutions not belonging to any of the cited (C). Completed CTs, with or without (w/out) posted results (D), completed CTs catalogued as Early phase/Phase I, as a single category, Phase II, Phase III, and Phase IV (E). Completed CTs according to funding resources as coming from industry, $\mathrm{NIH}$, Federal agencies other than $\mathrm{NIH}$, and Universities or other institutions not belonging to any of the cited $(\mathrm{F})$.

N/A, not available; NIH, National Institutes of Health.

due to overlaps when search filters are applied. Meaning that a study may commonly recruit participants of both sexes or indiscriminate older adult age or age in general. This must be considered when interpreting tabulated data of the supplementary files.

The country with the larger number of trials for the study of schizophrenia was USA, followed by Europe and China. Undeveloped countries reported the least number of trials. Surprisingly, developed countries with active research programs, large populations, and local pharmaceutical companies like Canada, Japan and Australia reported a relatively small number of studies, with only 280

\section{CTs $(<10 \%)$ in all three countries (Fig. 2).}

The fact that the number of CTs when adding all countries activities surpasses the total number of $\mathrm{CT}$ in schizophrenia (3,086 in total) indicates that many of the studies are multicenter and implicate international collaborations.

An additional filter to select among completed studies those reporting results was applied disappointedly finding that among the 1,929 completed studies, only $457(24 \%)$ reported results (Fig. 1A, D). Most of them (445) were interventional, with only 12 including observational objectives. Only $10 \%$ of them included participants on a pediatric age, and the age range $18-65$ was prevalent 


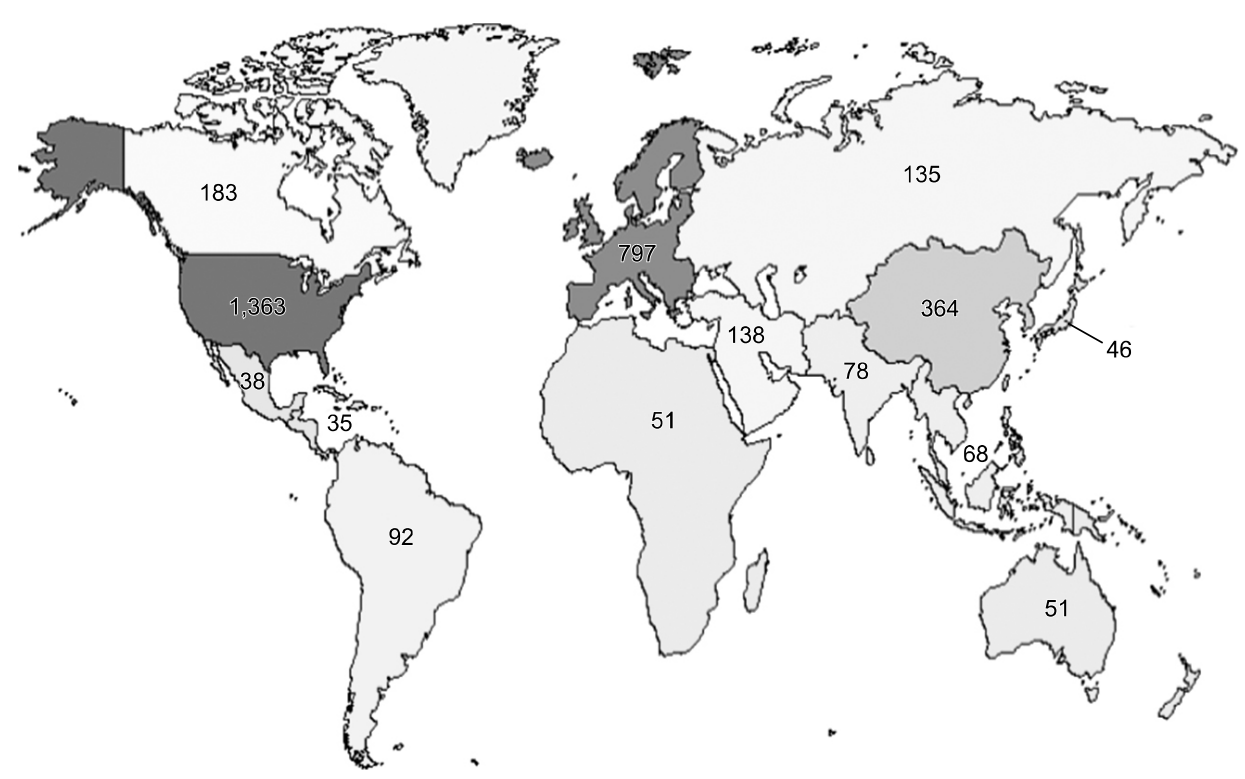

Fig. 2. Geographic distribution of clinical trials (CTs) for the study of schizophrenia according to participating countries. Countries appear grouped by geographic areas shown in different colors. The number of CTs for the study of schizophrenia for each geographic area appears indicated in a box.

above the older adult ( $>65$ ) population. No significant sex bias in the inclusion criteria was detected (Supplementary Table 2; available online). Of those 457 completed studies with results, most (69\%) corresponded to phases II or later, while only $8 \%$ of the studies (37) corresponded to early phases of development, further supporting a more mature phase rather than early phases or new developments (Fig. 1E). For the completed with results CTs, industry financing seems to have played a major role with over $50 \%$ of the initiatives, followed by universities and federal funds (Fig. 1E; Supplementary Table 2).

With the intention of evidencing the features of the CTs associating to current main treatments, a focused search for treatments in the categories of anti-psychotics, anti-depressants, dopamine antagonists and cognitive remediation therapy were performed. Analogously to the general search depicted above, search outputs corresponding to completed CTs with results were further classified by study status, eligibility criteria of participants (age and sex), study type, study phase, funder type and geographic location.

\section{CLINICAL TRIALS OF SCHIZOPHRENIA WITH ANTI-PSYCHOTICS}

The number of completed CTs evaluating the performance of anti-psychotics to treat schizophrenia was 1,451, with 1,015 completed and $286(<30 \%)$ reporting results (Fig. 3A; Supplementary Table 3; available online). Among the studies completed with reported results, only $8 \%$ corresponded to studies in early phase, while over $80 \%$ corresponded to more advanced phases (Fig. 3B). This indicates reduced new fronts of research in the area of this category of psychotropic compounds. It stands out the fact that main funders $(70 \%)$ of these studies were industries followed at a great distance by both, universities and federal resources (approximately 15\% contribution for each) (Fig. 3C; Supplementary Table 3).

Main implicated countries testing anti-psychotics were USA, followed by Europe and China. Developers of these compounds, like Japan, contributed to less extent to these trials (Supplementary Fig. 1; available online).

\section{CLINICAL TRIALS OF SCHIZOPHRENIA WITH ANTI-DEPRESSANTS}

In the case of anti-depressants, it was found that 431 CTs registered the assay of these compounds, with 302 being completed and 87 providing results $(<30 \%$ of the completed) (Fig. 4A). Almost all trials (99\%) in this category were interventional with only one study reporting observational design. A predominance age range of adults from 18 to 65 years over older adults and children (17\%) were reported. No sex bias was detected in these CTs ei- 
A Antipsychotic drug

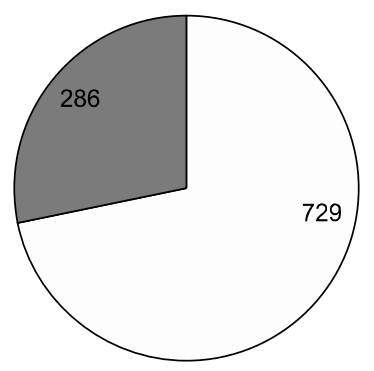

W/out results

\section{B}

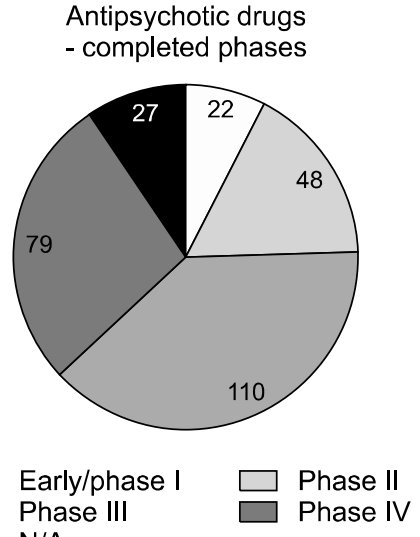

C
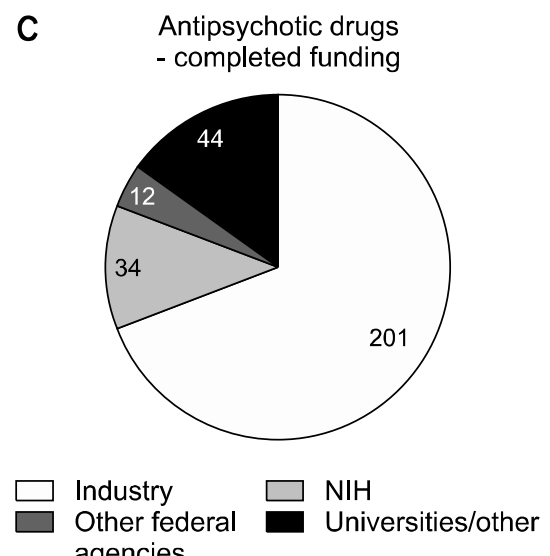

Fig. 3. Completed clinical trials (CTs) for the study of anti-psychotics in the treatment of schizophrenia. Completed CTs without (w/out) or with posted results are indicated (A); classification according to study phase, catalogued as Early phase/Phase I, as a single category, Phase II, Phase III and Phase IV. N/A corresponds to trials that have not been catalogued by study phase (B); and funding resources, classified as coming from industry, $\mathrm{NIH}$, Federal agencies other than $\mathrm{NIH}$, and Universities or other institutions not belonging to any of the cited (C).

$\mathrm{N} / \mathrm{A}$, not available; $\mathrm{NIH}$, National Institutes of Health.

A Antidepressants

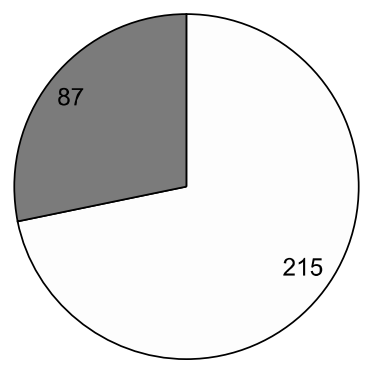

W/out results $\square$ With results
B
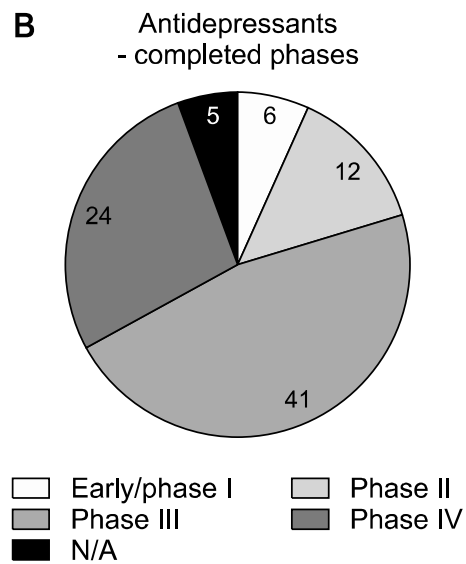

C
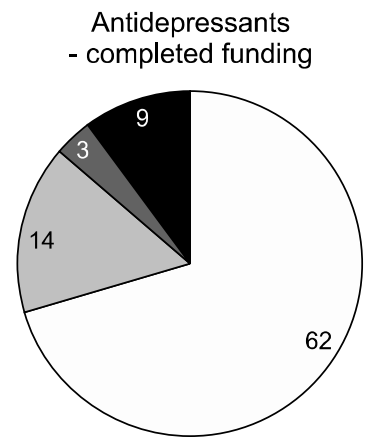

$\square$ Industry
$\begin{aligned} & \text { Other federal } \\ & \text { agencies }\end{aligned}$$\quad \begin{aligned} & \text { UIH } \\ & \text { Universities/other }\end{aligned}$

Fig. 4. Completed clinical trials (CTs) with reported results for the study of anti-depressants in the treatment of schizophrenia. Completed CTs without results (w/out results) or with them, are indicated (A); classification according to study phase, catalogued as Early phase/Phase I, as a single category, Phase II, Phase III and Phase IV. N/A corresponds to trials that have not been catalogued by study phase (B); and funding resources, classified as coming from industry, $\mathrm{NIH}$, Federal agencies other than $\mathrm{NIH}$, and Universities or other institutions not belonging to any of the cited (C). $\mathrm{N} / \mathrm{A}$, not available; NIH, National Institutes of Health.

ther (Supplementary Table 4; available online).

Early phases included a low number of CTs (7\%) in relation to more CTs at more advanced phases, indicative of low recent activity at the initial steps of drug testing also here (Fig. 4B). Finally, industry was the top funder of the research of this category of compounds ( $>70 \%$ ) at a great distance with federal funds (19\%) and the lowest participation of universities (10\%) (Fig. 4C; Supplementary Table 4).

The geographic distribution of CTs of schizophrenia with anti-depressants follow a similar trend as the described for antipsychotics, with USA leading in numbers, followed by Europe and China, and with Little participation of Canada, Japan and Australia (Supplementary Fig. 2; available online).

\section{CLINICAL TRIALS OF SCHIZOPHRENIA WITH DOPAMINE ANTAGONISTS}

In the category of dopamine antagonists, a total of 702 studies were found, among them, only 168 trials were completed with registered results (32\%) (Fig. 5A) while the rest of completed trials did not reported any. Most (99\%) were interventional and, as the other drug-based 
A

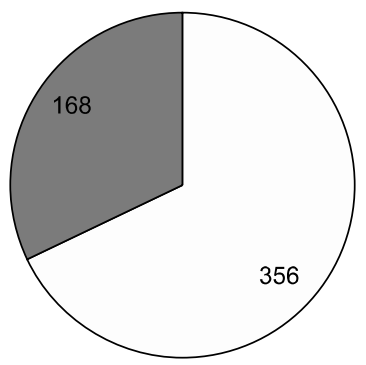

W/out results

\section{B}
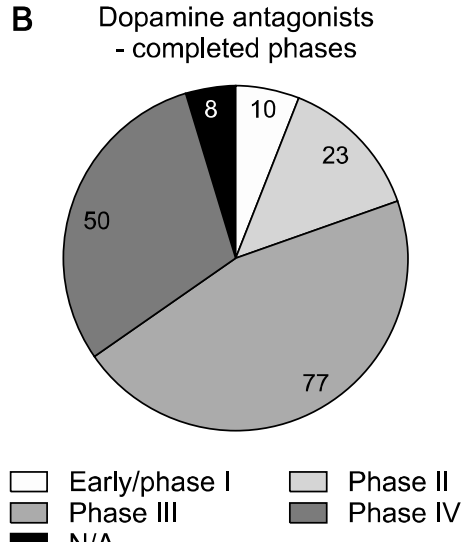
N/A

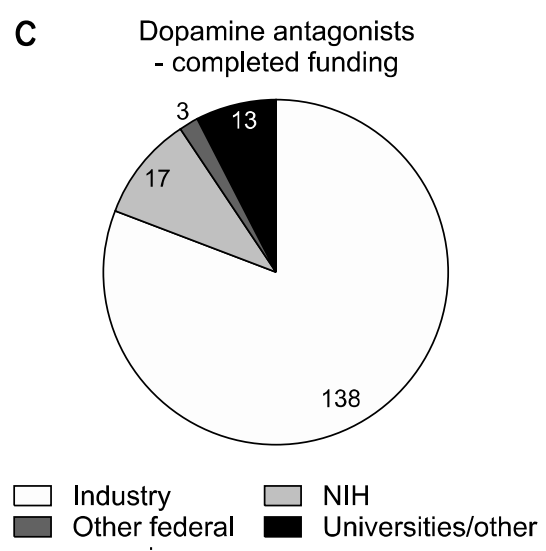

agencies

Fig. 5. Completed clinical trials (CTs) with reported results for the study of dopamine antagonists in the treatment of schizophrenia. Completed CTs without results (w/out results) or with results are indicated (A). Classification according to study phase, catalogued as Early phase/Phase I, as a single category, Phase II, Phase III and Phase IV. N/A corresponds to trials that have not been catalogued by study phase (B); and funding resources classified as coming from industry, $\mathrm{NIH}$, Federal agencies other than $\mathrm{NIH}$, and Universities or other institutions not belonging to any of the cited (C). $\mathrm{N} / \mathrm{A}$, not available; $\mathrm{NIH}$, National Institutes of Health.

trials from former sections, most included adults in the age range of $18-65$, followed by older adults and a smaller participation of pediatric patients (14\%). No sex bias was detected in these CTs either (Supplementary Table 5; available online). In this case, the early phase completed with results trials corresponded to $6 \%$ (10/ $168)$, thus, indicating an advanced stage of research with over $90 \%$ of CTs in phase II or later (Fig. 5B). Funding most notorious source in this category was industry in with over $80 \%$ of the overall funds and the lowest coming from universities (8\%) (Fig. 5C; Supplementary Table 5). As for the previous category of drugs, clinical research with these compounds presents low current activity despite the many unknowns separating responders from non-responders and the paucity of information on potential sex-and age- factors playing a role in therapy, or the options to minimize therapy secondary effects.

The geographic distribution of CTs of schizophrenia with dopamine antagonists follow a similar trend as the described for antipsychotics and anti-depressants, as expected, with USA leading in numbers, followed by Europe and China, and with Little participation of Canada, Japan and Australia (Supplementary Fig. 3; available online).

A limitation of the design presented here is that some compounds included in the group of antipsychotics, including risperidone, olanzapine, roflumilast, perphenazine and aripiprazole are also included in some CTs under the categories of anti-depressant and dopamine antagonists.

\section{CLINICAL TRIALS OF SCHIZOPHRENIA WITH COGNITIVE REMEDIATION THERAPY \& NATURAL COMPOUNDS}

Although Cognitive Behavioral Therapy (CBT) was found ineffective by Kennedy and Xyrichis [15], it was of interest to evaluate the efforts invested in this drug-free option for the treatment of schizophrenia as compared to pharmaceutical-based approaches.

A much lower number of CTs were found for this category (119) with a striking low participation of industry (only $4 / 119$ or $3 \%$ ) (Supplementary Table 6; available online). Only 74 studies had been completed, and among those, only $16 \%$ contained registered data (12/74) (Fig. 6A). All CTs completed with results studies appeared registered as interventional, and only one including pediatric participants (Supplementary Table 6). Only 2 of the 12 appeared in late phases of development (phases III and IV) (Fig. 6B), suggesting CBT may constitute a more recent initiative to explore alternative, drug-free methods to alleviate symptoms in schizophrenic patients. Contrary to drug-based CTs, none of the completed studies with results in this category were funded by the industry. Instead, federal funds led the initiatives in almost $70 \%$ of the cases (8/12) followed by universities (4/12) (Fig. 6C; Supplementary Table 6).

The geographic distribution of CTs of schizophrenia with CBT is also led by USA, only one study in Canada and another in Europe have been completed up to date in- 
A

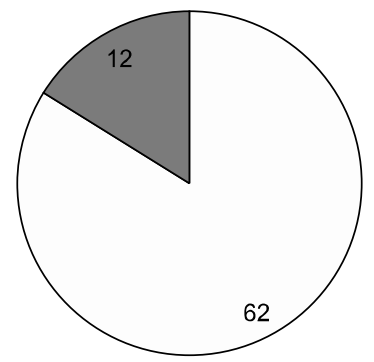

W/out results

\section{B}

Cognitive remediation - completed phases
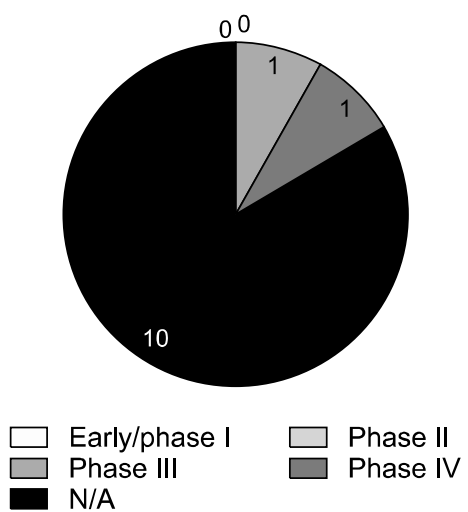

C
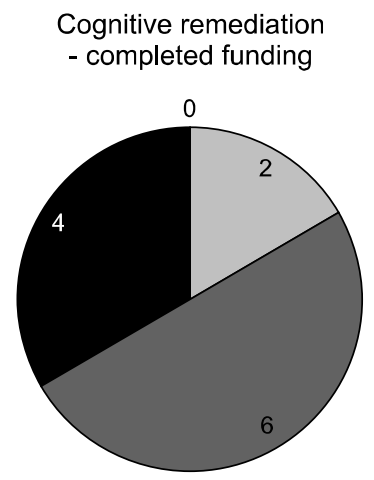

$\square$ Industry
$\begin{aligned} & \text { Other federal } \\ & \text { agencies }\end{aligned}$ $\begin{aligned} & \text { UIH } \\ & \text { Universities/other }\end{aligned}$

Fig. 6. Completed clinical trials (CTs) with reported results for the study of cognitive remediation therapy in the treatment of schizophrenia. Completed CTs without (w/out) or with posted results are indicated (A); classification according to study phase catalogued as Early phase/Phase I, as a single category, Phase II, Phase III and Phase IV. N/A corresponds to trials that have not been catalogued by study phase (B); and funding resources classified as coming from industry, $\mathrm{NIH}$, Federal agencies other than $\mathrm{NIH}$, and Universities or other institutions not belonging to any of the cited (C). N/A, not available; NIH, National Institutes of Health.

dicating low activity in the development of drug-free therapeutic options for the management of the disease (Supplementary Fig. 4; available online).

The fact that the number of CTs/country equal the total number of trials in this category (CBT completed and with results) suggests they consist of independent studies not involving international collaborations.

Interestingly, when all cognitive remediation trials are considered (119 CTs), the number of studies performed in Canada (14/119) reach an important percentage with almost $12 \%$ of studies, just behind USA (62/119) and Europe (34/119). China does not report any CT for this type of therapy (data not shown).

Other drug-free initiatives are the CTs exploring the therapeutic power of natural compounds, mainly focusing on their anti-inflammatory properties (Supplementary Table 7; available online). Although still quite scarce (only $42 \mathrm{CTs}$ ), already 17 have been completed. Examples of these CTs using natural compounds can be found among this category, as it is the case of trial identifier number: NCT01793935 testing the effects of extracts from the plant Withania somnifera containing several bioactive molecules (withanolides, withanosides, indosides, withaferin-A, others) with attributed immunomodulatory, anti-inflammatory and stress reducing properties. Earlier results indicate minimal side effects while providing some benefits [16].

A second example found was the $\mathrm{CT}$ with identification number: NCT02104752. A formulation with improved bioavailability of curcumin assessed by the neurogenesis marker brain derived nerve factor in addition to clinical symptoms [17], with the objective of improving symptoms by reducing inflammation.

Among the novel initiatives worth mentioning we found NCT02769936 Phase I trial using a single dose of D-cycloserine as an experimental probe to evaluate the effects of enhancing N-methyl-D-aspartate receptor (NMDAR)signaling on plasticity versus working memory in affected individuals; NCT00960219, evaluating the effects of benzoate, a D-amino acid oxidase inhibitor, as a method to enhance NMDA function [18]; NCT 01390376, determining the benefits of the addition of benzoate to the last-line antipsychotic agent for refractory schizophrenia clozapine [19]; and NCT01047592 using benzoate in combination with sarcosine, a glycine transporter I inhibitor acting as an adjuvant NMDA-enhancing agent [20].

As an overall, it seems that evaluation of natural approaches rather than testing of synthetic small molecules are preferred among new initiatives. However, they seem presently rather scarce in number (Supplementary Tables $6,7)$.

\section{FUTURE FOREFRONTS}

Although the term "schizophrenia" was coined about a century ago, a mental illness presenting psychotic dis- 
crete episodes was already described at the end of the XIX century by Kraepelin [21], the review of historic documents has posited the disease to the beginnings of mankind. With such a long history of clinical descriptions and recompilation of data, with findings that include brain anatomic deformities in monozygotic twins [22] and multiple neurological dysfunctions, it seems surprising that the disease still remains enigmatic at various levels. Not only its diagnosis stays poorly defined, currently relaying on unspecific clinical symptoms, but also controversies keep its origin under intense debate.

It is possible that subtypes of patients associating with different levels of progression or degree of affection in combination with multiple environmental factors difficult the identification of key players for the diagnosis. In fact, the International Society for Central Nervous System (CNS) Clinical Trials and Methodology Working Group states that perhaps it is a more realistic approach to identify patient subgroups sharing common biological patterns than try allocating a unique "schizophrenia biomarker" [23]. The limitations for diagnosis and thus the heterogeneity of participants in CTs possibly has translated into difficulties towards identification of effective treatments. In line with this concept, a recent review by Maslej et al. [24], highlights the relevance of personalizing schizophrenia treatments to contemplate individual response differences.

New technology might allow individualized studies overcoming previous limitations imposed by disease heterogeneity. The development of in vitro models including patient-derived induced pluripotent stem cells (iPSCs), brain organoid systems and human brain circuitry platforms will more faithfully reproduce true human disease scenarios compared to the artificial rodent and lower animal models available. Thus, they may help evaluate metabolic [25] and epigenetic associations [26] to further understand the multifactorial nature of schizophrenia and set subgroup criteria for patient subtyping. In addition, these systems might be key in exploring effective treatments of the disease by providing platforms for the screening of compounds in the affected pathways, at the individual level. The systems are capable of incorporating individual risk factors (DISC1, NRG1 and miR-137, and other), allelic variants of complement factor $\mathrm{C} 4$ genes and other that have been associated with excessive synaptic pruning [27] and that may vary across individuals. To re- duce unnecessary variance, however, identification of subgroups of patients presenting overlapping genotypes is recommended. Recent CNS model improvements have yielded the production of microglia, which are critical for the formation and maintenance of neural circuits, within brain organoids [28]. Also, it is contemplated that 3D-vascular network could be possibly added to brain organoids in a near future.

These individualized in vitro platforms should also allow to discern individual cross-reactivity susceptibility commonly derived from drug unspecific interactions, allowing improvement of results in future CTs. Dosage comparison or combination with additional compounds to minimize drug-secondary effects, as for example, omega fatty acid supplementation, most likely to prevent weight gain and increased cholesterol levels are also among future improvements.

In summary, the prevalence of CTs with results in advanced phases indicates that the research of the treatment of schizophrenia with psychotropic agents is not new. Novel initiatives are few and mainly focused on natural drug-free alternatives, perhaps allowing future therapeutic options with reduced side effects. A paucity of CTs to determine sex- and age- differences among patients is also noticed. On another side, it seems worrisome that drugfree alternatives might attract less attention by pharmaceutical investments and thus remain underdeveloped. Future hopes seem to rely on CTs based on novel model findings at the individual level allowing the development of precision medical programs towards effectively treating schizophrenia. Perhaps the mentioned models serve to develop self-healing programs to repair the neurogenesis defects found in post-mortem brains of schizophrenic individuals $[29,30]$.

\section{CONCLUSIONS}

- CTs have intensively explored the treatment of schizophrenia with over 3,000 studies performed from which almost 2/3 are completed. Investigational designs were predominant over observational, most including adult, sex-unbiased populations.

- The reduced numbers of ongoing (active) and early phase/phase I CTs indicate low research activity in new forefronts for the treatment of the disease.

- Surprisingly, developed countries with active research 
programs, large populations, and local pharmaceutical companies producers of schizophrenia drug treatments, including Canada, Japan and Australia reported relatively small numbers of CTs $(<10 \%)$ compared to USA and Europe (> 70\%).

- The features of CTs for the three type of pharmacological treatments selected (anti-psychotic, anti-depressants and dopamine antagonists) fit with the trends of the completed trials in general, reaching, in the case of the selected treatments, up to $80 \%$ of funding resources from industry. By contrast, the non-pharmacological approach reviewed (cognitive remediation or $\mathrm{CBT}$ ) included very low numbers of trials (74 CTs) and among the studies completed with results none were funded by industry.

- The attributed anti-inflammatory and immune-modulatory properties of curcumin and extracts from the Indian plant Withania somnifera are being explored in CTs for treatment of schizophrenia. Only one of the 17 completed CTs has been sponsored by industry.

- CTs with compound combinations to reduce druginduced secondary effects are ongoing.

- Future drug-free focused research, including CBT, life style control and the use of natural compounds to prevent and/or treat schizophrenia symptoms are urgently needed.

- iPSCs derived from patients, brain organoid systems and human brain circuitry platforms are now available as promising models for the study of the disease. Future treatment trials relying on personalized, precision optimized programs perhaps oriented to potentiate neurogenesis and thus self-healing, are warranted.

\section{Acknowledgments}

The author would like to thank Dr. Elisa Oltra and Dr. Ignacio Ventura for critically reviewing the manuscript.

\section{Conflicts of Interest}

No potential conflict of interest relevant to this article was reported.

\section{REFERENCES}

1. Cowen P, Harrison P, Burns T. Shorter oxford textbook of psychiatry. 6th ed. Oxford:Oxford University Press;2012.

2. Schizophrenia Commission. The abandoned illness: a report by the Schizophrenia Commission. London:Rethink Mental IIIness;2012.

3. Cloutier M, Aigbogun MS, Guerin A, Nitulescu R, Ramanakumar $\mathrm{AV}$, Kamat SA, et al. The economic burden of schizophrenia in the United States in 2013. J Clin Psychiatry 2016;77:764-771.

4. Owen MJ, Sawa A, Mortensen PB. Schizophrenia. Lancet 2016;388:86-97.

5. Carter CJ. Schizophrenia susceptibility genes directly implicated in the life cycles of pathogens: cytomegalovirus, influenza, herpes simplex, rubella, and Toxoplasma gondii. Schizophr Bull 2009;35:1163-1182.

6. Murray RM, Mehta M, Di Forti M. Different dopaminergic abnormalities underlie cannabis dependence and cannabis-induced psychosis. Biol Psychiatry 2014;75:430-431.

7. Yang AC, Tsai SJ. New targets for schizophrenia treatment beyond the dopamine hypothesis. Int J Mol Sci 2017;18:1689.

8. Lin $\mathrm{CH}$, Lane HY. Early identification and intervention of schizophrenia: insight from hypotheses of g/utamate dysfunction and oxidative stress. Front Psychiatry 2019;10:93.

9. Rodrigues-Amorim D, Rivera-Baltanás T, Spuch C, Caruncho HJ, González-Fernandez Á, Olivares JM, et al. Cytokines dysregulation in schizophrenia: a systematic review of psychoneuroimmune relationship. Schizophr Res 2018;197:19-33.

10. Fernandes BS, Steiner J, Bernstein HG, Dodd S, Pasco JA, Dean OM, et al. C-reactive protein is increased in schizophrenia but is not altered by antipsychotics: meta-analysis and implications. Mol Psychiatry 2016;21:554-564.

11. Bertolote J, McGorry P. Early intervention and recovery for young people with early psychosis: consensus statement. Br J Psychiatry Suppl 2005;48:s116-s119.

12. Keating D, McWilliams S, Schneider I, Hynes C, Cousins G, Strawbridge J, et al. Pharmacological guidelines for schizophrenia: a systematic review and comparison of recommendations for the first episode. BMJ Open 2017;7:e013881.

13. National Institute for Health and Care Excellence (UK). 2019 exceptional surveillance of psychosis and schizophrenia in adults: prevention and management (NICE guideline CG178) [Internet]. London: National Institute for Health and Care Excellence (UK); 2019 Mar 12 [cite at 2020 Jan 30]. Available from: http://www.ncbi.n/m.nih.gov/books/NBK551816/.

14. U.S. National Library of Medicine. ClinicalTrials.gov [Internet]. Bethesda: U.S. National Library of Medicine [cited at 2019 Jun 28]. Available from: https://clinicaltrials.gov/.

15. Kennedy L, Xyrichis A. Cognitive behavioral therapy compared with non-specialized therapy for alleviating the effect of auditory hallucinations in people with reoccurring schizophrenia: a systematic review and meta-analysis. Community Ment Health J 2017;53:127-133.

16. Chengappa KNR, Brar JS, Gannon JM, Schlicht PJ. Adjunctive use of a standardized extract of Withania somnifera (Ashwagandha) to treat symptom exacerbation in schizophrenia: a randomized, double-blind, placebo-controlled study. J Clin Psychiatry 2018;79:17m11826. 
17. Wynn JK, Green MF, Hellemann G, Karunaratne K, Davis MC, Marder SR. The effects of curcumin on brain-derived neurotrophic factor and cognition in schizophrenia: a randomized controlled study. Schizophr Res 2018;195:572-573.

18. Lane HY, Lin CH, Green MF, Hellemann G, Huang CC, Chen PW, et al. Add-on treatment of benzoate for schizophrenia: a randomized, double-blind, placebo-controlled trial of D-amino acid oxidase inhibitor. JAMA Psychiatry 2013;70:1267-1275.

19. Lin $\mathrm{CH}$, Lin $\mathrm{CH}$, Chang YC, Huang YJ, Chen PW, Yang HT, et al. Sodium benzoate, a D-amino acid oxidase inhibitor, added to clozapine for the treatment of schizophrenia: a random ized, double-blind, placebo-controlled trial. Biol Psychiatry 2018;84:422-432.

20. Lin CY, Liang SY, Chang YC, Ting SY, Kao CL, Wu YH, et al. Adjunctive sarcosine plus benzoate improved cognitive function in chronic schizophrenia patients with constant clinical symptoms: a randomised, double-blind, placebo-controlled trial. World J Biol Psychiatry 2017;18:357-368.

21. Kraepelin E. [Psychiatrie: ein lehrbuch für studirende und aerzte]. 5th ed. Leipzig:J.A. Barth;1896. German.

22. Reveley AM, Reveley MA, Clifford CA, Murray RM. Cerebral ventricular size in twins discordant for schizophrenia. Lancet 1982;1:540-541.

23. Goff DC, Romero K, Paul J, Mercedes Perez-Rodriguez M, Crandall D, Potkin SG. Biomarkers for drug development in early psychosis: current issues and promising directions. Eur Neuropsychopharmacol 2016;26:923-937.
24. Maslej MM, Furukawa TA, Cipriani A, Andrews PW, Mulsant $\mathrm{BH}$. Individual differences in response to antidepressants: a meta-analysis of placebo-controlled randomized clinical trials. JAMA Psychiatry 2020;e194815.

25. Rajasekaran A, Venkatasubramanian G, Berk M, Debnath M. Mitochondrial dysfunction in schizophrenia: pathways, mechanisms and implications. Neurosci Biobehav Rev 2015; 48:10-21.

26. Föcking M, Doyle B, Munawar N, Dillon ET, Cotter D, Cagney G. Epigenetic factors in schizophrenia: mechanisms and experimental approaches. Mol Neuropsychiatry 2019;5:6-12.

27. Cannon TD, Chung Y, He G, Sun D, Jacobson A, van Erp TGM, et al. Progressive reduction in cortical thickness as psychosis develops: a multisite longitudinal neuroimaging study of youth at elevated clinical risk. Biol Psychiatry 2015;77: 147-157.

28. Ormel PR, Vieira de Sá $R$, van Bodegraven EJ, Karst $H$, Harschnitz O, Sneeboer MAM, et al. Microglia innately develop within cerebral organoids. Nat Commun 2018;9:4167.

29. Tamminga CA, Stan AD, Wagner AD. The hippocampal formation in schizophrenia. Am J Psychiatry 2010;167:11781193.

30. Walton NM, Zhou Y, Kogan JH, Shin R, Webster M, Gross AK, et al. Detection of an immature dentate gyrus feature in human schizophrenia/bipolar patients. Transl Psychiatry 2012; 2:e135. 